\title{
Radiation-induced pulmonary injury accelerated pulmonary metastasis in a mouse model of breast cancer
}

\author{
HONG-YUN GONG, WEI-GUO HU, QIN-YONG HU, XIANG-PAN LI and QI-BIN SONG \\ Department of Oncology, Cancer Center, Renmin Hospital of Wuhan University, Wuhan, Hubei 430060, P.R. China
}

Received October 28, 2014; Accepted August 25, 2015

DOI: $10.3892 / 01.2015 .3810$

\begin{abstract}
The aim of the present study was to investigate the acceleration of pulmonary metastasis due to pulmonary injury caused by radiation treatment in a mouse model of breast cancer, in addition to determining the associated mechanism. The passive metastatic breast cancer model was used in radiation-treated $\mathrm{BALB} / \mathrm{c}$ mice. In total, 24 mice were randomly separated into two groups, with 12 mice per group, and the groups were treated with or without pulmonary radiation. The survival time and variation of the weights of the lungs, spleen and liver were recorded. Lung metastasis was also evaluated, and chemokine (C-X-C motif) ligand 12 (CXCL12)/chemokine (C-X-C motif) receptor 4 (CXCR4) expression was determined. The results revealed that the group with radiation-induced pulmonary injury exhibited an increased incidence of pulmonary metastasis and shorter survival time compared with the mice without pulmonary radiation. The radiation-treated group possessed an increased number of metastatic nodules in the lungs, but metastasis was not evident in the liver and spleen. The CXCL12/CXCR4 axis was markedly expressed and the expression was significantly increased subsequent to radiation compared with the expression in normal lung tissues. The present study demonstrated that radiation-induced pulmonary injury may accelerate metastatic tumor growth and decrease the overall survival rate of the mice following in situ injection of tumor cells. Tumor localization and growth may have been favored by metastatic conditioning in the lung subsequent to radiotherapy. The CXCL12/CXCR4 axis may affect key elements in the multistep process of metastasis induced by radiation injury.
\end{abstract}

\footnotetext{
Correspondence to: Professor Qi-Bin Song, Department of Oncology, Cancer Center, Renmin Hospital of Wuhan University, 238 Jiefang Road, Wuhan, Hubei 430060, P.R. China

E-mail: qibin_song@sina.com
}

Key words: CXCL12/CXCR4, radiation, pulmonary injury, metastasis

\section{Introduction}

Radiation has previously been the main therapy in tumor treatment, and may prolong the survival time of patients by improving the local control rate of tumors (1). However, for patients with breast, esophageal, pulmonary and mediastinal lymph node cancer masses treated by radiation, radiation-induced pulmonary injury frequently occurs $(2,3)$. Patients may develop sub-acute pneumonitis or late fibrosis subsequent to radiation exposure, which are the most common complications of radiotherapy that result in eventual mortality (3).

Following the treatment of patients by radiation, acute inflammation may be resolved with the recruitment of fibroblasts, resulting in interstitial collagen deposition and alveolar septal thickening during alveolar epithelial regeneration (4). Endothelial dysfunction is mainly caused by vascular damage and endothelial barrier injury, which may activate various pathophysiological cascades (5). Blood plasma permeates the damaged vascular barrier, and affects structural elements of vessels by activating the specific receptors (6). This change in tissue homeostasis may, in turn, lead to a chronic inflammatory response that does not subside (7). Radiation has previously been found to change the cell phenotype and tumor microenvironment, and as a result, an increased number of invasive residual tumor cells demonstrated higher rates of metastasis (8). However, the pathophysiology of radiation induced pneumonitis is complicated and remains unclear, although studies have indicated that inflammatory mediators affect genetic stability and cause persistent epigenetic alteration $(9,10)$. This indicates that inflammatory components of the tumor microenvironment affect fundamental mechanisms responsible for the generation of metastatic variants (11). Therefore, it was hypothesized that radiation-induced pulmonary injury may accelerate metastasis in the cancer patients that received chest radiation.

In the present study, the impact of radiation-induced pulmonary injury on the lung metastasis of breast cancer was examined in mice. Furthermore, the present study aimed to reveal the mechanism by which radiation-induced pulmonary injury accelerated the lung metastasis of breast cancer, and it has been hypothesized that there may be a novel method to prevent radiation-induced pulmonary injury and metastasis subsequent to radiotherapy. 


\section{Materials and methods}

In vivo experiments. A total of $24 \mathrm{BALB} / \mathrm{c}$ inbred strains of female mice (age, 4-6 weeks; weight, 16-18 g) were purchased from the Experimental Animal Center of Wuhan University (Wuhan, Hubei, China). All animal experiments were approved by the Scientific Ethics Committee of Renmin Hospital of Wuhan University (approval no. KF 01-143/03; Wuhan, Hubei, China). The animals were bred in a barrier-free animal house in the First Clinical College of Wuhan University Laboratory Animal Center (Wuhan, Hubei, China). All mice were housed in accordance with the Guide for the Care and Use of Laboratory Animals (12).

The mice were randomly divided into the radiation and control groups, with 12 mice in each group. To generate the mammary cancer model, the mice in each group were injected with $3 \times 10^{5}$ mammary carcinoma 4T1 tumor cells (American Type Culture Collection, Manassas, VA, USA) in the right last mammary gland. For thoracic irradiation, the mice were anesthetized by intraabdominal administration of chloral hydrate (dose, $400 \mathrm{mg} / \mathrm{kg}$ in mice). The right chest in the radiation group was irradiated by $6 \mathrm{MV} \mathrm{X}$-rays from a linear accelerator (LINAC ; Elekta Oncology Systems, Ltd., Crawley, UK), at a dose of 9 Gy when tumors grew to measurable sizes, ranging between 3 and $5 \mathrm{~mm}$ in diameter, 7 days subsequent to tumor cell transplantation. The size of the treatment field was $1.3 \times 0.8 \mathrm{~cm}$.

The body weights of the mice were tested every day and the survival rate was also evaluated. Following irradiation, spontaneous lung metastasis was scored every week. The mice were sacrificed (three/week, every week) and the lungs were removed for weighing. Metastasis was assessed by observing the appearance of the lung, and the white round nodules on the surface of the yellowish lung were counted as metastatic lesions. The regions of the lungs were separated and fixed in buffered formaldehyde (Tianjin Kermel Chemical Reagent Co., Ltd, Tianjin,China) for immunohistochemical analysis.

Immunohistochemistry for detecting the chemokine (C-X-C motif) ligand 12 (CXCL12)/chemokine (C-X-C motif) receptor 4 (CXCR4) axis. At the indicated times subsequent to irradiation, 3 or 4 mice from each group were sacrificed by cervical dislocation, 1, 2, 3 or 4 weeks following irradiation. Sections of the lungs were formalin-fixed and paraffin-embedded. Coronal sections of the lung were sliced into $5-\mathrm{mm}$ sections, and the slides with the largest cross section were stained with hematoxylin and eosin. The slides were evaluated by a pathologist without knowledge of the treatment administered. Immunohistochemical analysis of CXCL12 and CXCR4 expression was performed according to the routine protocol (13). Briefly, 4-5- $\mu \mathrm{m}$ sections were de-paraffinized in xylene and rehydrated through serial solutions of ethanol, consisting of $95,90,80$ and $70 \%$ ethanol. Subsequent to antigen retrieval and blockage of endogenous peroxidase activity, the sections were incubated with polyclonal mouse or rabbit anti-CXCL12 (\#ab18919; 1:1,000; Abcam, Cambridge, UK) and monoclonal anti-CXCR4 (\#ab124824; 1:1,000; Abcam) antibodies at $4^{\circ} \mathrm{C}$ for $8-12 \mathrm{~h}$, followed by detection using 3,3'-diaminobenzidine coloration.
Statistical analysis. Statistical analysis was performed using SigmaStat software (Jandel Scientific, San Rafael, CA, USA). All results are presented as the mean \pm standard deviation of at least six independent experiments. The data of the groups were compared using non-paired $t$-test. $\mathrm{P}<0.05$ was considered to indicate a statistically significant difference.

\section{Results}

Development of lethality following thoracic irradiation. Firstly, the effect of various doses of radiation on the mice was investigated. Following radiation treatment at a dose of $9 \mathrm{~Gy}$, the mice did not succumb to pneumonitis, but succumbed, in general, 4-5 weeks later due to metastasis. In addition, sub-acute pneumonitis occurred at 2 weeks after irradiation and progressively increased over the next 2 weeks. Lung metastasis began to appear at 3 weeks. Finally, the possibility that radiation-induced pulmonary injury may decrease the survival time in mice was investigated. A passive metastatic model was used in the radiation-treated BALB/c mice, and the mice that were intrahepatically injected with $4 \mathrm{~T} 1$ cells 7 days subsequent to radiation treatment demonstrated a shorter median survival time of 21 days, compared with 27 days in the mice without radiation treatment. The survival time of the radiation-treated mice was significantly shorter compared with the survival time of untreated mice (Fig. 1). In addition, the effect on survival was reflected in a statistically significant reduction in body weight in the radiation-treated mice compared with untreated mice ( $\mathrm{P}<0.05$; Fig. 2).

Metastasis formation. It was hypothesized that the mice with radiation-induced pulmonary injury may possess an increased risk of lung metastasis and associated cancer progression, which may contribute to the shorter survival time in radiation-treated mice. To test this hypothesis, mice were sacrificed weekly subsequent to the administration of radiation. Lung metastatic progression was evaluated by measuring the lung wet weight, counting the lung nodules and histological examination. All mice that succumbed demonstrated an extensive tumor burden in the lungs, but evident metastasis was not observed in other organs, including the liver and spleen, indicating that the mice succumbed to lung metastasis (Fig. 3). Histological examination revealed the infiltration of inflammatory cells in the early period subsequent to radiation and the presence of lung metastatic nodes at 3 weeks (Fig. 4). The present results provide evidence that the mice with radiation-induced pulmonary injury may demonstrate an increased risk of lung metastasis.

Expression of the CXCL12/CXCR4 axis in lung tissues of mice. The contribution of the levels of the CXCL12/CXCR4 axis to the metastatic activity of mammary carcinoma $4 \mathrm{~T} 1$ cells was investigated. It has been reported that the activation of the CXCL12/CXCR4 axis was important in the development of radiation-induced pulmonary fibrosis (14). The CXCL12/CXCR4 axis participated in the vascularization induced by radiation and was closely associated with the recurrence and metastasis of breast cancer subsequent to radiation. In the present study, the expression level of CXCL12/CXCR4 in treated mice increased markedly following radiation and was significantly increased compared 


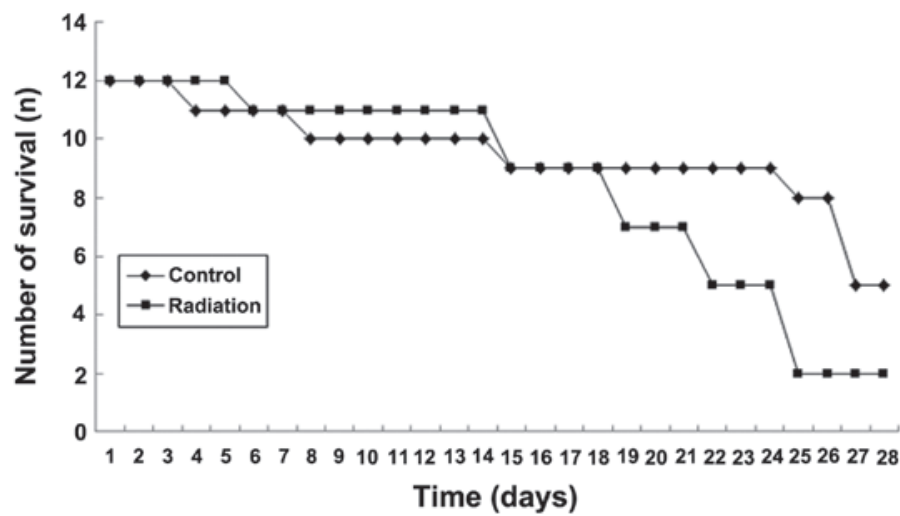

Figure 1. Survival time of mice treated with and without radiotherapy. The survival time of radiation-treated mice ( $\mathrm{n}=12)$ was significantly shorter compared with the survival time of the untreated mice $\left(\mathrm{n}=12,{ }^{*} \mathrm{P}<0.05\right)$.

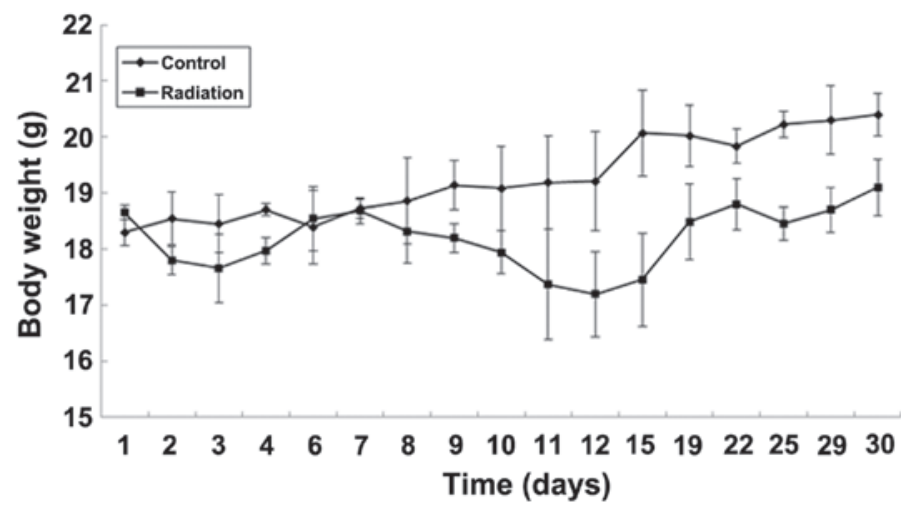

Figure 2. Changes in the body weight of mice treated with and without radiotherapy. The weight loss of radiation-treated mice was evident. In the first 3 days, weight loss may be associated with acute radiation injury. In days 12-30, lung metastasis may have been responsible for the weight loss. At the end of the study, the in situ tumor demonstrated extremely rapid growth, and therefore the weight was increasing.
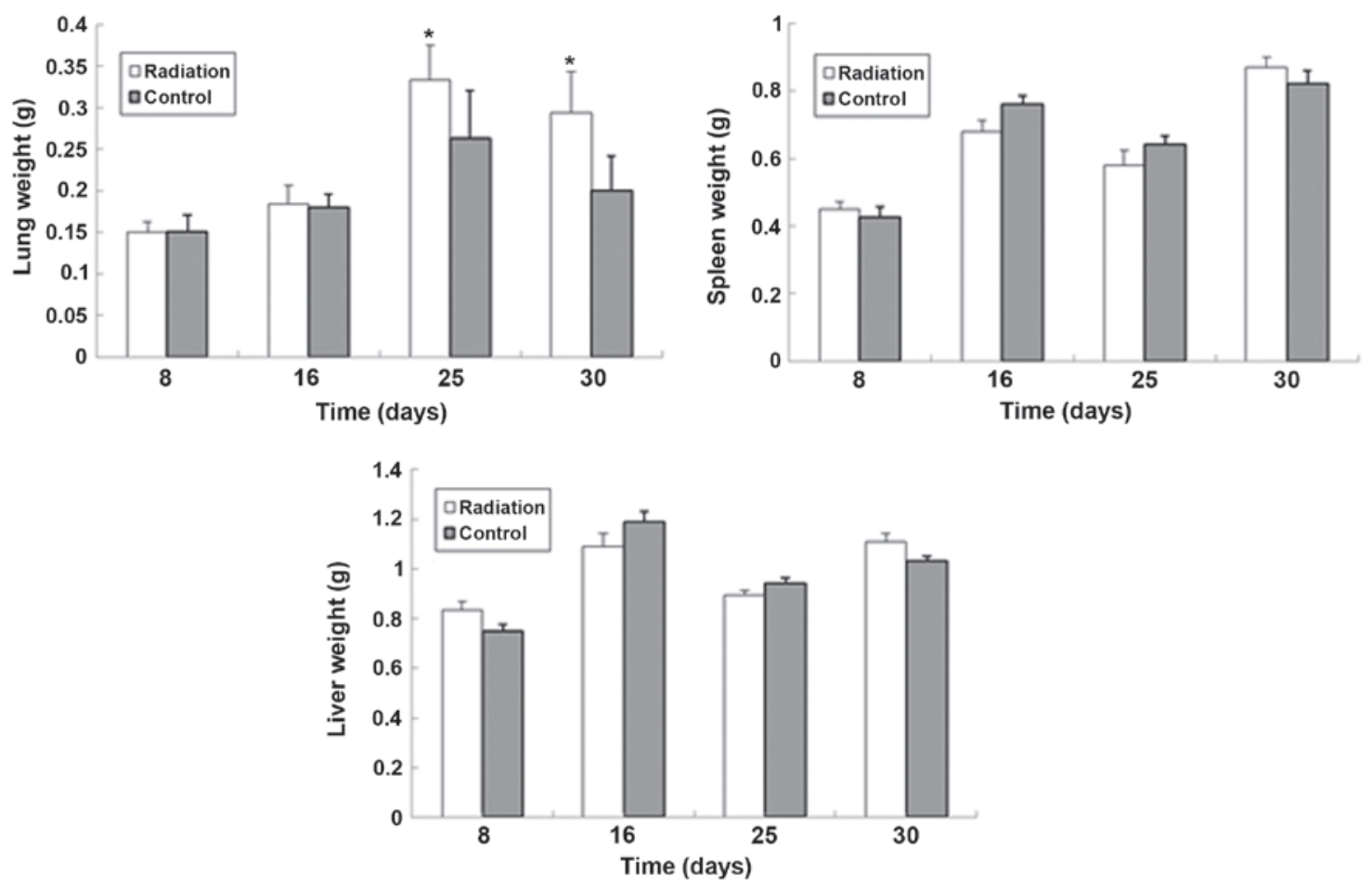

Figure 3. Weight variation of the lung, spleen and liver. Compared with the spleen and liver, the weight of the lungs in radiation-treated mice was not different from the untreated weight in the first two weeks, but in the later two weeks, the lung weight of mice increased further subsequent to lung radiation. This may be associated with lung metastasis." $\mathrm{P}<0.05$. 

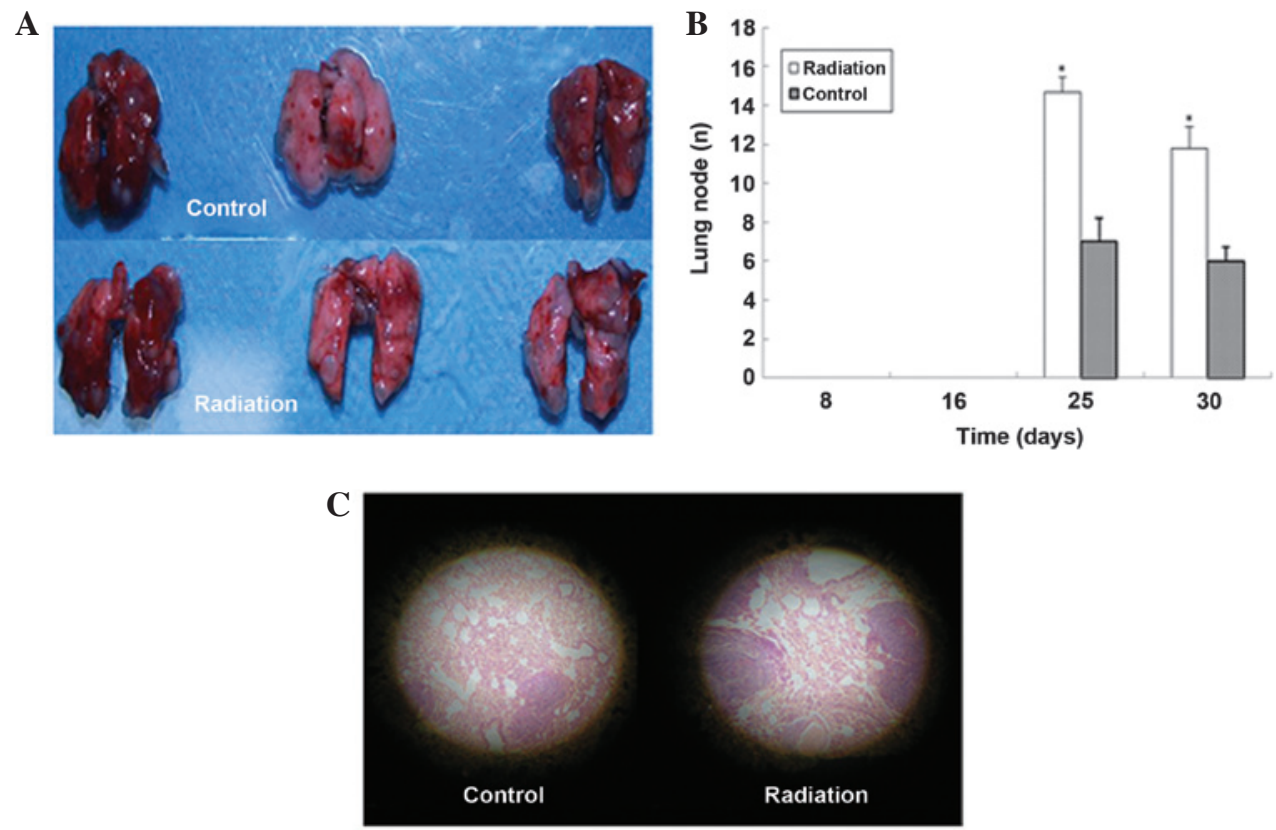

Figure 4. Lung node and hematoxylin and eosin pathological section. (A) The lung nodes of radiation-treated mice were increased in size compared with the nodes of untreated mice. (B) Comparison between the lung nodes of radiation-treated and control mice. ${ }^{~} \mathrm{P}<0.05$. (C) In the same field of view, an increased number of nodes were identified in the radiation-treated mice.

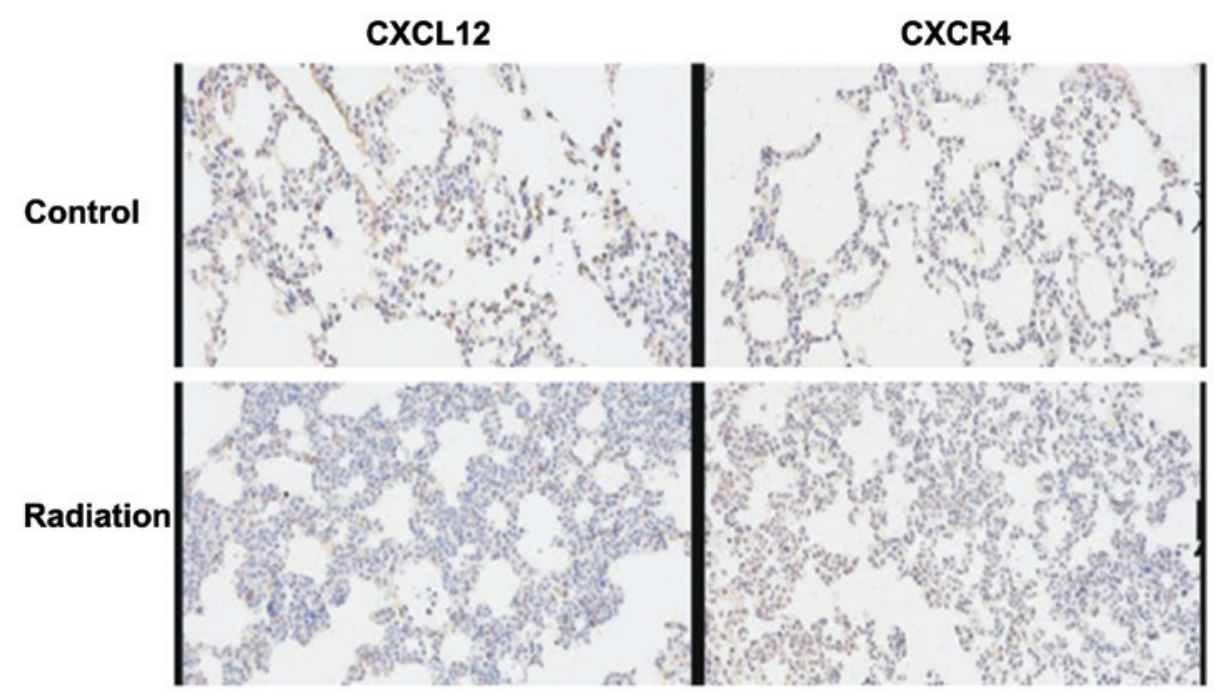

Figure 5. Expression of the CXCL12/CXCR4 axis in the lung tissues of mice. The expression of CXCL12/CXCR4 in the treated mice markedly increased and was significantly increased compared with normal lung tissues ( $\mathrm{P}<0.05)$. CXCL12, chemokine (C-X-C motif) ligand 12; CXCR4, chemokine (C-X-C motif) receptor 4 .

with normal lung tissues (Fig. 5). The present results indicate that the CXCL12/CXCR4 axis was associated with pulmonary metastasis accelerated by radiation-induced pulmonary injury.

\section{Discussion}

To the best of our knowledge, the present study demonstrated for the first time that radiation-induced pulmonary injury may accelerate pulmonary metastasis in a passive metastatic breast cancer BALB/c mouse model. This finding is supported by studies that have revealed that irradiated fibroblasts accelerate the invasive growth of non-irradiated adenocarcinoma cells $(15,16)$. The aforementioned irradiated lung-induced phenomena were elicited through a bystander mechanism involving the interaction of cancer and normal cells, as the tumor itself was not irradiated. This indicated that the effects of radiation on cancer and normal cells should be considered in cancer radiotherapy. In the present study, the precise underlying mechanism of the aforementioned radiation-induced pulmonary metastasis phenomena was unclear. It was hypothesized that the activation of surrounding stromal cells and recruitment of various inflammatory cells subsequent to radiation was involved in the multistep process of invasion and metastasis. In addition to promoting carcinogenesis, myelomonocytic cells and the mediators of these cells affect the key components of the multistep process of metastasis, including the interaction with the extracellular matrix and the construction of a pre-metastatic niche (17). 
Radiation-induced lung injury may be divided into two phases, consisting of an acute inflammatory phase and a late fibrotic phase $(18,19)$. The histopathological changes to the irradiated lung have been well described $(18,20,21)$. The inflammatory phase is generally characterized by alveolar cell depletion and inflammatory cell accumulation $(4,22)$. This process initiated the activation of specific leukocyte subsets to produce important biological mediators, including cytokines, growth factors and chemokines, which participate in the majority of the aspects of the inflammatory response (4). Bone marrow derived cells are chemotactically recruited to sites of radiation tissue injury and fibroblasts are directly involve in the fibrotic pathway. The fibrotic phase includes fibroblast proliferation, collagen accumulation and alveolar septal thickening (22). It was hypothesized that the acute inflammation during the early phase lead to the later fibrosis phase, but a direct and causal association between early acute inflammation and the later fibrotic phase has not been established $(23,24)$. The recruitment and activation of monocytes, macrophages and lymphocytes is a key component of radiation-induced lung injury, and chemokines are important mediators in the pathogenesis of lung injury in several environments $(25,26)$. Previous studies have reported that the expression of chemokines and chemokine receptors is elevated in tumor cells, fibrosis-sensitive mice and patients that have undergone radiotherapy (27-29). In addition, blockade of the chemokine ligand and the associated receptors may prevent lung inflammation and fibrosis in $\mathrm{C} 57 \mathrm{BL} / 6 \mathrm{~J}$ mice that received thoracic radiation (30). Therefore, it was hypothesized that the expression of specific chemokines and chemokine receptors during the acute inflammatory phase induced by radiation may result in the recruitment and activation of lymphocytes and macrophages, which then contribute to the late fibrotic phase.

It has previously been reported that activation of the chemotactic receptor CXCR4 by the ligand CXCL12 was important in the development of radiation-induced pulmonary fibrosis (14). Subsequent to radiation-induced injury, bone marrow-derived fibroblast progenitor cells, also termed fibrocytes, which express CXCR4, are recruited to regions of fibrosis in the lung $(25,31)$. A neutralizing antibody against CXCL12 may prevent the recruitment of circulating fibrocytes to radiation-damaged lung and suppress the development of fibrosis. The CXCL12-CXCR4 axis was also reported to participate in the vascularization induced by radiation (32), in addition to being closely associated with recurrence and metastasis subsequent to radiation $(33,34)$. Previous studies have demonstrated that stromal fibroblast fractions extracted from a number of invasive human breast carcinomas were more able to promote the growth of mammary carcinoma cells and to enhance tumor angiogenesis compared with the comparable cells derived from outside of these tumor masses $(35,36)$. Furthermore, high concentrations of CXCL12 gradients in the lung, liver and lymph nodes more easily attracted circulating CXCR4-expressing tumor cells to such sites (37). These findings are not unique to breast cancer and were also found in other types of tumors (38-42). According to previous findings, the CXCL12/CXCR4 axis participates in the pathophysiological process of metastasis in at least 23 types of tumors (43). High levels of the CXCR4 expression by various types of human carcinoma cells are clinically associated with a poor prognosis (44). Therefore, it was hypothesized that the CXCL12/CXCR4 axis played an important role in the acceleration of the metastasis of breast cancer due to radiation. As a result, the expression levels of CXCL12/CXCR4 were tested in treated mice, and were found to be increased subsequent to radiation, compared with normal lung tissues. To a certain extent, this result confirmed the present hypothesis.

In summary, radiation-induced pulmonary injury leads to a chronic inflammatory response, which produces an eligible pre-metastatic microenvironment for cancer cells. It is possible that the CXCL12/CXCR4 axis affects key elements in the multistep process of invasion and metastasis. However, additional studies are required to validate the exact mechanism.

\section{Acknowledgements}

The authors thank the doctors and nurses of the Department of Oncology, Cancer Center, Renmin Hospital of Wuhan University for their prodigious support.

\section{References}

1. Jeremic B, Fidarova E, Sharma V, Faheem M, Ameira AA, Nasr Ben Ammar C, Frobe A, Lau F, Brincat S and Jones G: The International Atomic Energy Agency (IAEA) randomized trial of palliative treatment of incurable locally advanced non-small cell lung cancer (NSCLC) using radiotherapy (RT) and chemotherapy (CHT) in limited resource setting. Radiother Oncol 116: 21-26, 2015

2. Abid SH, Malhotra V and Perry MC: Radiation-induced and chemotherapy-induced pulmonary injury. Curr Opin Oncol 13: 242-248, 2001

3. Mehta V: Radiation pneumonitis and pulmonary fibrosis in non-small-cell lung cancer: pulmonary function, prediction, and prevention. Int J Radiat Oncol Biol Phys 63: 5-24, 2005.

4. Tsoutsou PG and Koukourakis MI: Radiation pneumonitis and fibrosis: Mechanisms underlying its pathogenesis and implications for future research. Int J Radiat Oncol Biol Phys 66: 1281-1293, 2006

5. Ghafoori P, Marks LB, Vujaskovic Z and Kelsey CR: Radiation-induced lung injury. Assessment, management and prevention. Oncology (Williston Park) 22: 37-47, discussion 52-53, 2008.

6. Alphonsus CS and Rodseth RN: The endothelial glycocalyx: A review of the vascular barrier. Anaesthesia 69: 777-784, 2014.

7. Li X, Xue J and Lu Y: Current situation and prospect of treatment for radiation-induced lung injury. Sheng Wu Yi Xue Gong Cheng Xue Za Zhi 27: 937-940, 2010 (In Chinese).

8. Shin JW, Son JY, Raghavendran HR, Chung WK, Kim HG, Park HJ, Jang SS and Son CG: High-dose ionizing radiation-induced hematotoxicity and metastasis in mice model. Clin Exp Metastasis 28: 803-810, 2011.

9. Kim JY, Kim YS, Kim YK, Park HJ, Kim SJ, Kang JH, Wang YP, Jang HS, Lee SN and Yoon SC: The TGF- $\beta 1$ dynamics during radiation therapy and its correlation to symptomatic radiation pneumonitis in lung cancer patients. Radiat Oncol 4: 59, 2009.

10. Jarnicki A, Putoczki T and Ernst M: Stat3: Linking inflammation to epithelial cancer - more than a "gut" feeling? Cell Div 5: 14, 2010.

11. Solinas G, Marchesi F, Garlanda C, Mantovani A and Allavena P: Inflammation-mediated promotion of invasion and metastasis. Cancer Metastasis Rev 29: 243-248, 2010.

12. Commission on Life Sciences National Research Council: Guide for the care and use of laboratory animals. The National Academies Press, Washington, DC, 1996.

13. Li T, Li H, Wang Y, Harvard C, Tan JL, Au A, Xu Z, Jablons DM and You L: The expression of CXCR4, CXCL12 and CXCR7 in malignant pleural mesothelioma. J Pathol 223: 519-530, 2011

14. Shu HK, Yoon Y, Hong S, Xu K, Gao H, Hao C, Torres-Gonzalez E, Nayra C, Rojas $M$ and Shim H: Inhibition of the CXCL12/CXCR4-Axis as preventive therapy for radiation-induced pulmonary fibrosis. Plos One 8: e79768, 2013. 
15. Barcellos-Hoff MH and Ravani SA: Irradiated mammary gland stroma promotes the expression of tumorigenic potential by unirradiated epithelial cells. Cancer Res 60: 1254-1260, 2000.

16. Ohuchida K, Mizumoto K, Murakami M, Qian LW, Sato N, Nagai E, Matsumoto K, Nakamura T and Tanaka M: Radiation to stromal fibroblasts increases invasiveness of pancreatic cancer cells through tumor-stromal interactions. Cancer Res 64: 3215-3222, 2004.

17. Sofia Vala I, Martins LR, Imaizumi N, Nunes RJ, Rino J, Kuonen F, Carvalho LM, Rüegg C, Grillo IM, Barata JT, et al: Low doses of ionizing radiation promote tumor growth and metastasis by enhancing angiogenesis. PLoS One 5: e11222, 2010.

18. Movsas B, Raffin TA, Epstein AH and Link CJ Jr: Pulmonary radiation injury. Chest 111: 1061-1076, 1997.

19. Xie L, Zhou J, Zhang S, Chen Q, Lai R, Ding W, Song C, Meng X and $\mathrm{Wu}$ J: Integrating microRNA and mRNA expression profiles in response to radiation-induced injury in rat lung. Radiat Oncol 9: 111, 2014.

20. Libshitz HI: Radiation changes in the lung. Semin Roentgenol 28 : 303-320, 1993.

21. Chou CH, Teng CM, Tzen, KY, Chang YC, Chen JH and Cheng JC: MMP-9 from sublethally irradiated tumor promotes Lewis lung carcinoma cell invasiveness and pulmonary metastasis. Oncogene 31: 458-468, 2012.

22. Morgan GW and Breit SN: Radiation and the lung: A reevaluation of the mechanisms mediating pulmonary injury. Int J Radiat Oncol Biol Phys 31: 361-369, 1995.

23. Ward PA and Hunninghake GW: Lung inflammation and fibrosis. Am J Respir Crit Care Med 157: S123-S129, 1998.

24. Johnston CJ, Williams JP, Elder A, Hernady E and Finkelstein JN Inflammatory cell recruitment following thoracic irradiation. Exp Lung Res 30: 369-382, 2004.

25. Phillips RJ, Burdick MD, Hong K, Lutz MA, Murray LA, Xue YY, Belperio JA, Keane MP and Strieter RM: Circulating fibrocytes traffic to the lungs in response to CXCL12 and mediate fibrosis. J Clin Invest 114: 438-446, 2004.

26. Heinzelmann F, Jendrossek V, Lauber K, Nowak K, Eldh T, Boras R, Handrick R, Henkel M, Martin C, Uhlig S, et al: Irradiation-induced pneumonitis mediated by the CD95/CD95-ligand system. J Natl Cancer Inst 98: 1248-1251, 2006.

27. Satoko M and Sandra D: Up-regulation of the Pro-inflammatory chemokine CXCL16 is a common response of tumor cells to ionizing radiation. Radiat Res 173: 418-425, 2010.

28. Johnston CJ, Williams JP, Okunieff $P$ and Finkelstein JN: Radiation-induced pulmonary fibrosis: Examination of chemokine and chemokinereceptor families. Radiat Res 157: 256-265, 2002

29. Okera M, Bae K, Bernstein E, Cheng L, Lawton C, Wolkov H, Pollack A, Dicker A, Sandler H and Sweeney CJ: Evaluation of nuclear factor $\kappa \mathrm{B}$ and chemokine receptor CXCR4 co-expression in patients with prostate cancer in the radiation therapy oncology group (RTOG) 8610. BJU Int 108: E51-E58, 2011.

30. Yang X, Walton W, Cook DN, Hua X, Tilley S, Haskell CA, Horuk R, Blackstock AW and Kirby SL: The chemokine, CCL3 and its receptor, CCR1, mediate thoracic radiation-induced pulmonary fibrosis. Am J Respir Cell Mol Biol 45: 127-135, 2011.
31. Andersson-Sjöland A, de Alba CG, Nihlberg K, Becerril C, Ramírez R, Pardo A, Westergren-Thorsson G and Selman M: Fibrocytes are a potential source of lung fibroblasts in idiopathic pulmonary fibrosis. Int J Biochem Cell Biol 40: 2129-2140, 2008.

32. Zong ZW, Cheng TM, Su YP, Ran XZ, Shen Y, Li N, Ai GP, Dong SW and Xu H: Recruitment of transplanted dermal multipotent stem cells to sites of injury in rats with combined radiation and wound injury by interaction of SDF-1 and CXCR4. Radiat Res 170: 444-450, 2008

33. Balkwill F: Cancer and the chemokine network. Nat Rev Cancer 4: 540-550, 2004

34. Kulbe H, Levinson NR, Balkwill F and Wilson JL: The chemokine network in cancer-much more than directing cell movement. Int J Dev Biol 48: 489-496, 2004.

35. Orimo A and Weinberg RA: Stromal fibroblasts in cancer: A novel tumor-promoting cell type. Cell Cycle 5: 1597-1601, 2006.

36. Orimo A, Gupta PB, Sgroi DC, Arenzana-Seisdedos F, Delaunay T, Naeem R, Carey VJ, Richardson AL and Weinberg RA: Stromal fibroblasts present in invasive human breast carcinomas promote tumor growth and angiogenesis through elevated SDF-1/CXCL12 secretion. Cell 121: 335-348, 2005.

37. Muller A, Homey B, Soto H, Ge N, Catron D, Buchanan ME, McClanahan T, Murphy E, Yuan W, Wagner SN, et al: Involvement of chemokine receptors in breast cancer metastasis. Nature 410: 50-56, 2001.

38. Phillips RJ, Burdick MD, Lutz M, Belperio JA, Keane MP and Strieter RM: The stromal derived factor-1/CXCL12-CXC chemokine receptor 4 biological axis in non-small cell lung cancer metastases. Am J Respir Crit Care Med 167: 1676-1686, 2003.

39. Scotton CJ, Wilson JL, Scott K, Stamp G, Wilbanks GD, Fricker S, Bridger G and Balkwill FR: Multiple actions of the chemokine CXCL12 on epithelial tumor cells in human ovarian cancer. Cancer Res 62: 5930-5938, 2002.

40. Rubin JB, Kung AL, Klein RS, Chan JA, Sun Y, Schmidt K, Kieram MW, Luster AD and Segal RA: A small-molecule antagonist of CXCR4 inhibits intracranial growth of primary brain tumors. PNAS 100: 13513-13518, 2003.

41. Bajetto A, Barbieri F, Dorcaratto A, Barbero S, Daga A, Porcile C, Ravetti JL, Zona G, Spaziante R, Corte G, Schettini G and Florio T: Expression of CXC chemokine receptor 1-5 and their ligands in human glioma tissues: role of CXCR4 and SDF1 in glioma cell proliferation and migration. Neurochem Int 49: 423-432, 2006

42. Scala S, Ottaiano A, Ascierto PA, Cavalli M, Simeone E, Giuliano P, Napolitano M, Franco R, Botti G and Castello G: Expression of CXCR4 predicts poor prognosis in patients with malignant melanoma. Clin Cancer Res 11: 1835-1841, 2005.

43. Balkwill F: Chemokine biology in cancer. Semin Immunol 15: 49-55, 2003.

44. Staller P, Sulitkova J, Lisztwan J, Moch H, Oakeley EJ and Krek W: Chemokine receptor CXCR4 downregulated by von hippel-lindau tumour suppressor pVHL. Nature 425: 307-311, 2003. 